\title{
Double-Gradient DGGE for Optimized Detection of DNA Point Mutations
}

BioTechniques 22:326-330 (February 1997)

\author{
L. Cremonesi, S. Firpo, M. \\ Ferrari, P.G. Righetti ${ }^{1}$ and \\ C. Gelfi ${ }^{2}$ \\ I.R.C.C.S., H. San Raffaele, \\ Milan, ${ }^{1}$ Università di Verona, \\ Faculty of Science, Verona and \\ 2ITBA, CNR, Milan, Italy
}

\section{INTRODUCTION}

Detection and localization of singlebase differences in specific regions of genomic DNA are of primary importance in the analysis of mutations associated with human diseases. Among the various screening methods (for a review see Reference 13), one technique is emerging for its sensitivity, accuracy, speed and manipulation simplicity: denaturing gradient gel electrophoresis (DGGE) $(9,19)$ and its variant, temperature gradient gel electrophoresis (TGGE) (20). It consists of co-amplifying the wild-type (Wt) and presumptive mutant (M) DNAs so as to obtain, prior to analysis, a family of fragments: two hetero- and two homo-duplexes. Separation among the various members of the family will not occur when electrophoresis is performed in the absence of denaturants: the minute differences in radius of gyration $(\mathrm{Rg})$, due to the mismatch, between the two hetero- and homo-duplexes will only lead, at best, to band broadening. However, if the same set of duplexes is run against a gradient of denaturants (either chemical or thermal), each duplex will be intercepted along the migration path by an isoperichoric milieu in which the environmental conditions will match exactly the melting temperature $\left(\mathrm{T}_{\mathrm{m}}\right)$ of its lowest melting domain. This domain will suddenly be destabilized and a transition from an orderly helix to a partially unwound DNA molecule will take place, leading to strong retardation in the gel due to markedly increased frictional drag with the gel matrix and the solvent molecules. Due to small, but non-negligible, differences in $\mathrm{T}_{\mathrm{m}}$ values of the various duplexes, a spectrum of four bands is typically resolved, indicating the presence of a point mutation along a DNA filament. Small shifts in $\mathrm{T}_{\mathrm{m}}$ of the corresponding melting domain may be due to transversions (AT $\rightarrow$ TA, GC $\rightarrow$ CG) or mutations at the very end of cooperative melting domains, whereas switches of $\mathrm{G}: \mathrm{C}$ pairs to A:T pairs and vice versa, small deletions or insertions, or multiple mutations normally lead to larger shifts in $\mathrm{T}_{\mathrm{m}}$.

Structure denaturation by a heat treatment or denaturing solvents is qualitatively similar, although helixcoil transitions of double-stranded nucleic acids, as elicited by temperature gradients, can be better described quantitatively by statistical thermodynamics. Notwithstanding the more cumbersome experimental setup (often requiring the use of two thermostats for generating the temperature gradient and the use of special precautions for avoiding unequal solvent evaporation from the gel slab), TGGE is also gaining in popularity, as witnessed by bursts of reports on this methodology (17, $18,24,25)$. A similar method in a capillary format is being developed (11). However, for ease of manipulations and simplicity, DGGE seems to be much more popular; although it, too, suffers from some inconveniences. For instance, since the denaturant gradient slope and running times vary for every 
DNA region to be analyzed, different conditions have to be experimentally found and optimized, greatly affecting the routine application of the method. In addition, since often the running time needed for resolving the homo-duplexes greatly exceeds that needed for separating the hetero-duplexes, the latter often produce smears and fuzzy bands, indistinguishable from the background fluorescence after ethidium bromide staining. It occurred to us that, if DGGE were to be performed in the presence of a second gradient, able to suppress band broadening till the very end of the analysis, one might obtain markedly improved patterns. A typical gradient able to minimize band broadening is a porosity gradient, as typically utilized in native electrophoresis of complex protein mixtures or under denaturing conditions, in the presence of sodium dodecyl sulfate (12). We report here the application of this double (porosity and denaturant) gradient for detection of DNA point mutations with much improved banding patterns. This marked improvement comes with minimal extra experimental burden, since the double gradient still requires the use of the same, single, two-vessel gradient mixer utilized for pouring the standard DGGE slab.

\section{MATERIALS AND METHODS}

\section{DNA Specimens}

Double gradient (DG)-DGGE was applied for the detection of known mutations in the cystic fibrosis transmembrane conductance regulator (CFTR) gene and was compared with the standard DGGE technique. DNA specimens were obtained from cystic fibrosis (CF) patients. The following mutations and polymorphisms were analyzed: $\mathrm{M} 1 \mathrm{~V}(\mathrm{~A} \rightarrow \mathrm{G}$ transversion at position 133) (2) and $125 \mathrm{G} / \mathrm{C}(6)$ in exon 1; 1078delT (3), R352Q (1187 $\mathrm{G} \rightarrow \mathrm{A})(4)$, T338I (1145 C $\rightarrow \mathrm{T})(23)$, $\mathrm{R} 347 \mathrm{P}(1172 \mathrm{G} \rightarrow \mathrm{C})(7)$ and $\mathrm{R} 334 \mathrm{~W}$ $(1132 \mathrm{C} \rightarrow \mathrm{T})(10)$ in exon 7 ; 1717$1 \mathrm{G} \rightarrow \mathrm{A}(14), \mathrm{G} 542 \mathrm{X}(\mathrm{G} \rightarrow \mathrm{T}$ at 1756$)$ (16), 1784delG (8) and R553X (1789 $\mathrm{C} \rightarrow \mathrm{T})(5)$ in exon 11 ; and $\mathrm{S} 1251 \mathrm{~N}$ $(3384 \mathrm{G} \rightarrow \mathrm{A})(15)$ in exon 20 . DGGE conditions, including GC-clamped primer sequences, denaturant gradient, time and voltage of the electrophoretic separation were as already described (1). For increased resolution, heteroduplexes were generated at the end of the polymerase chain reaction (PCR) by 5 -min denaturation at $95^{\circ} \mathrm{C}$, followed by $1-\mathrm{h}$ reannealing at $55^{\circ} \mathrm{C}$.

\section{Gradient Gel Electrophoresis}

This technique was applied to every possible denaturing gradient slope as suggested by Myers et al. (19) (10\%$60 \%, 20 \%-70 \%, 30 \%-80 \%$ and $40 \%-$ $90 \%$ denaturant). Standard DGGE for all exons was performed at a constant $6.5 \% \mathrm{~T}$ polyacrylamide (in TEA buffer: $40 \mathrm{mM}$ Tris, $20 \mathrm{mM} \mathrm{NaOH}, 1 \mathrm{mM}$ EDTA and acetic acid added to $\mathrm{pH}$ 7.6) at the following specific conditions for each exon: exon 11: 10\%-60\% denaturant, $160 \mathrm{~V}, 5 \mathrm{~h}$; exon 20: $20 \%-70 \%$ denaturant, $160 \mathrm{~V}, 5 \mathrm{~h}$; exon 7: $30 \%-$ $80 \%$ denaturant, $75 \mathrm{~V}, 15 \mathrm{~h}$; and exon 1: 40\%-90\% denaturant, $160 \mathrm{~V}, 4 \mathrm{~h}$.

In the case of DG-DGGE, the same samples were analyzed in a $6.5 \%-12 \%$ $\mathrm{T}$ polyacrylamide gradient gel in the same TEA buffer and in the presence of the same gradients of denaturants as used for standard DGGE. For all samples, however, a single set of running

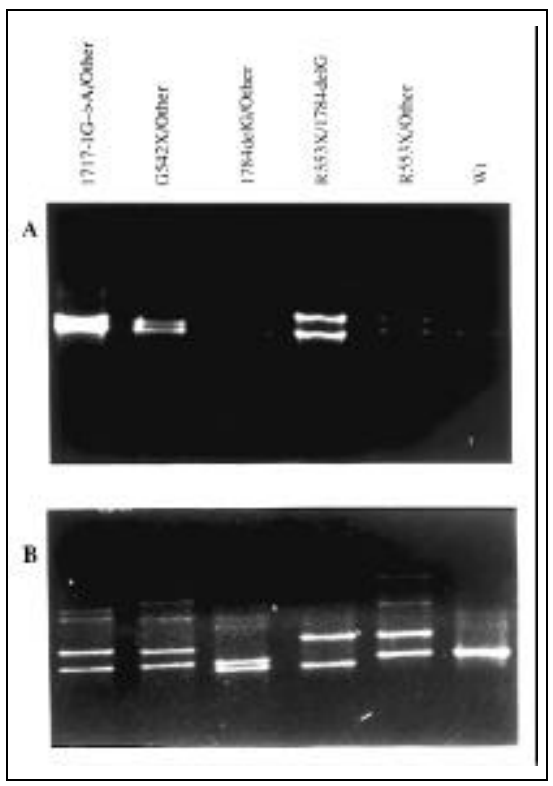

Figure 1. Comparison between DGGE (A) and DG-DGGE (B) for analysis of point mutations in exon 11 of the CFTR gene. Samples: 1, 1717$1 \mathrm{G} \rightarrow \mathrm{A} /$ other; 2, G542X/other; 3, 1784delG/other; 4, R553X/1784delG; 5, R553X/other; 6, Wt (wild-type). Denaturing gradient: 10\%-60\%. conditions was adopted: $75 \mathrm{~V}$ for $15 \mathrm{~h}$. The gel slabs were 0.75 -mm-thick, 15 $\mathrm{cm}$-wide and 16-cm-long. At the end of the run, the gels were stained in ethidium bromide.

\section{RESULTS}

Figure 1 gives a comparison of analysis of five mutations in exon 11 of the CFTR gene, as screened by DGGE (Panel A) and by DG-DGGE in a 10\%-60\% denaturing gradient (Panel B). It is immediately apparent that, in the former case, whereas the two homo-duplexes are resolved and quite visible, the two hetero-duplexes (which should appear more cathodically, since they are the first species to partial unwind) are barely visible, since they are smeared over a longer migration track. Conversely, in the case of DG-DGGE, the set of four bands is clearly visible in all tracks, and all bands are much

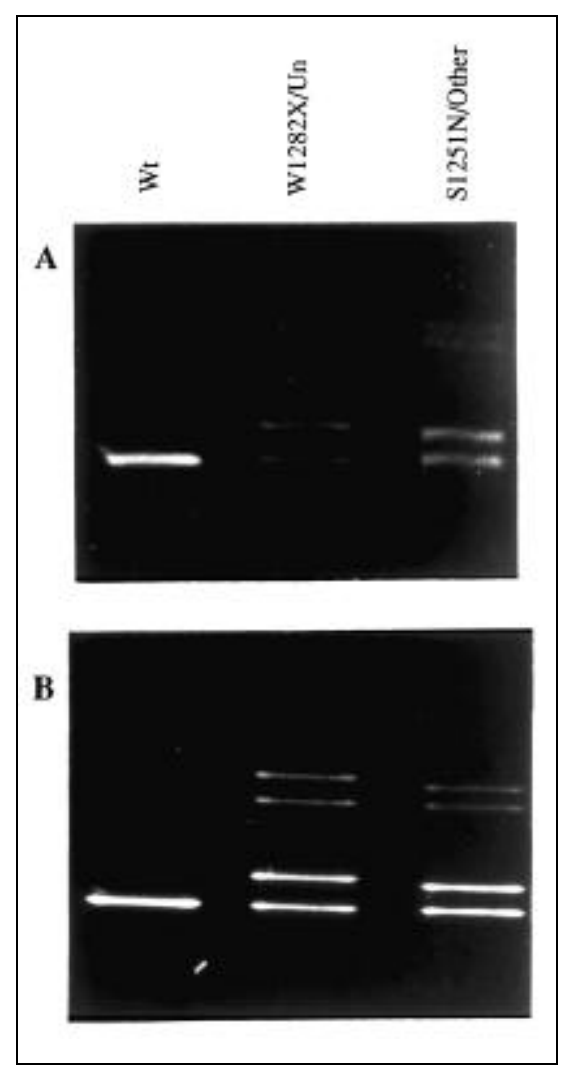

Figure 2. Comparison between DGGE (A) and DG-DGGE (B) for analysis of point mutations in exon 20 of the CFTR gene. Samples: 1, Wt; 2, W1282X/other; 3, S1251N/other. Denaturing gradient: $20 \%-70 \%$. 
sharper than in the corresponding DGGE gel. Moreover, concerning lanes 1 and 2, only DG-DGGE allows one to differentiate the specific patterns displayed by the $1717-1 \mathrm{G} \rightarrow \mathrm{A}$ and G542X mutations, which give almost identical patterns in conventional DGGE. An extra benefit is apparent when examining lane 3: in this case, not only are the two hetero-duplexes lost, but even the set of two homo-duplexes, due to minute $\mathrm{T}_{\mathrm{m}}$ values, seems to run as a single band. Under these conditions, this mutation might not be detectable, since the pattern resembles that of the wild-type (lane 6). However, the same sample in the DG-DGGE mode shows a clear splitting into two bands in the homoduplex zone.

Figure 2 shows the same comparison in the analysis of two mutations in exon 20 of the CFTR gene in a $20 \%-70 \%$ denaturing gradient. Here, too, the set of four bands in the DGDGGE is visible at a glance, whereas the standard DGGE gives fuzzy and diffused zones.

Figure 3 offers the same comparison in the analysis of five mutations in exon 7 of the CFTR gene in a $30 \%-80 \%$ denaturing gradient. This exon, which contains a large variety of mild mutations, is particularly difficult to analyze by conventional DGGE due to its base composition, as evident in Panel A. Here, too, the difference between conventional DGGE and DG-DGGE is quite remarkable.

Finally, Figure 4 explores two samples with the highest melting points in the CFTR gene: two mutations in exon 1 , requiring a $40 \%-90 \%$ denaturing gradient. Here, too, the marked pattern difference in polymorphism 125G/C (lane 3) (a G:C conservative transversion) should be appreciated: the two homo-duplexes are not resolved at all in DGGE, whereas DG-DGGE shows clearly a very close doublet of bands.

\section{DISCUSSION}

Given the above findings, and the superior results, it is surprising that the DG-DGGE technique has not been reported before. It is seen here that not only is the pattern of the two hetero-duplexes markedly improved, but even the set of homo-duplexes often offers superior patterns. The reasons for these marked improvements can be found in the following observations. First of all, it is known that, in the $5 \%$ to $15 \% \mathrm{~T}$ polyacrylamide gradient, the average pore radius of the matrix decreases rapidly in an exponential fashion (22). Thus, markedly increased friction is experienced by the analytes even along a reduced migration path in the anodic direction. This results in a higher band resolution, since migration through a medium of increased viscosity sharpens the zone by retarding the front more than the rear of the band, thus counteracting diffusion effects. Secondly, it is not unreasonable to think that the various bands are approaching the pore limit, where migration tends to cease asymptotically, since the band sharpening is truly remarkable. Some of the patterns obtained are similar to those obtained routinely by isoelectric focusing in immobilized $\mathrm{pH}$ gradients, where band sharpening is a result of the interplay of two antagonistic forces: entropic ones, tending to spread the analyte zone in the surrounding space, and "focusing" forces, driven by the high voltage gradient, tending to concentrate the sample in a very thin zone (21).

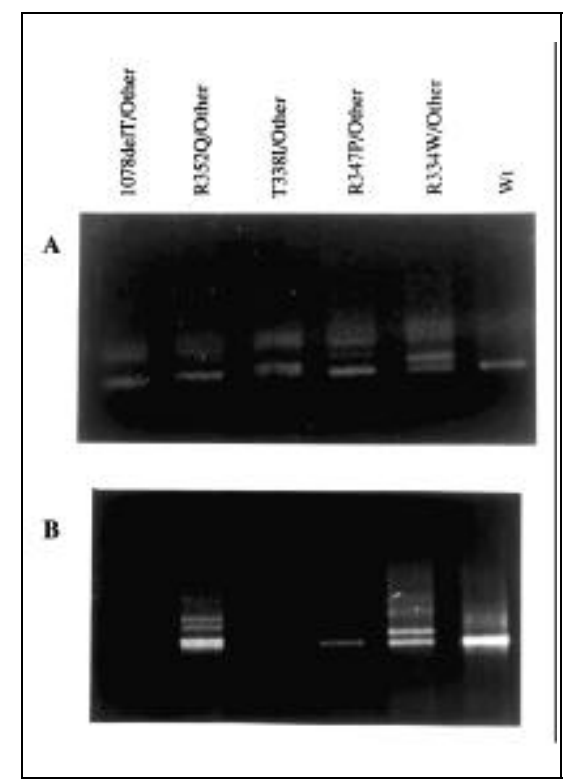

Figure 3. Comparison between DGGE (A) and DG-DGGE (B) for analysis of point mutations in exon 7 of the CFTR gene. Samples: 1, 1078delT/other; 2, R352Q/other; 3, T3381/other; 4, R347P/other; 5, R334W/other; 6, Wt. Denaturing gradient: $30 \%-80 \%$.
As stated above, this effect is more pronounced on the two hetero-duplexes which, under normal running conditions, are spread into a curtain of molecules, rather than offering a single banding zone. This might be due to the following. Hetero-duplexes, due to mismatch in some regions of the double helix, are much less resistant to denaturation and are the first molecules to partially unwind along the migration track. However, electrophoresis cannot be stopped at this point, since the set of two homo-duplexes has not developed as yet. As the train of zones progresses into regions of higher denaturant concentrations, needed for optimally resolving the two homo-duplexes, the two hetero-duplexes experience overdenaturing conditions, leading to progressive melting of the whole domain, which causes a strong decrease in mobility and consequently high diffusion of the banding zone. In presence of a porosity gradient, given a prolonged electrophoretic time, the set of mole-

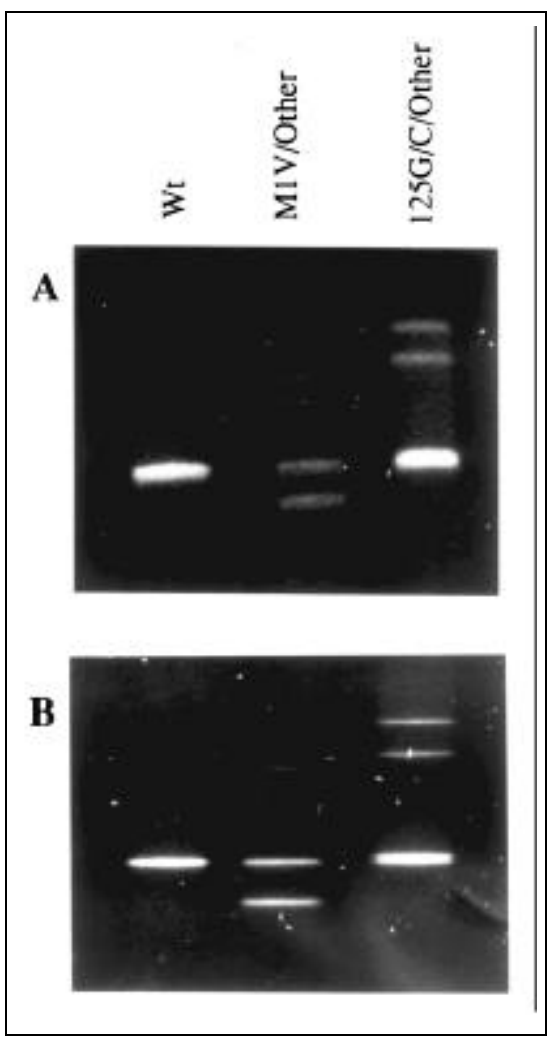

Figure 4. Comparison between DGGE (A) and DG-DGGE (B) for analysis of point mutations in exon 1 of the CFTR gene. Samples: $1, \mathrm{Wt} ; 2$, MIV/other; 3, 125G/C/other. Denaturing gradient: $40 \%-90 \%$. 
cules trailing behind have sufficient time to catch the front and re-compact the zone.

The improved band resolution allows a precise assignment of each electrophoretic pattern to a specific mutation, enhancing the diagnostic power of the technique. With the term diagnostic power, we imply the ability of our system to detect a known mutation present in a given sample on the basis of the migration identity, as compared with a known mutant migrating side by side on the same gel. This system is thus a valid screening test for identifying a panel of mutations comprising the molecular defects most frequent in a given population. The presence of a given mutation, for the direct diagnosis of a disease or of a linkage polymorphism with a given disease, to be used in indirect diagnosis can be further confirmed with restriction analysis or reverse dot blot and does not require direct or indirect sequencing of the amplified fragment. The DG-DGGE system proposed here is, nevertheless, valid also as a method for searching for new mutations; in this case, however, it should be further corroborated by sequencing for identifying point mutations. Especially in the case of genetic diseases such as cystic fibrosis, which is caused by more than 500 mutations, most of them described in a single case (CF Genetic Analysis Consortium), spread all over the gene, it is extremely important to have a highly sensitive technique that allows the identification of almost $100 \%$ of the mutations. A preliminary DG-DGGE screening for mutations that are predominant in a given population would allow their identification on the basis of the electrophoretic pattern compared with a set of known mutated control samples. Only samples negative for predominant mutations should be scanned for the whole coding region of the gene.

In conventional DGGE, different voltage gradients and running times have to be optimized according to the type of mutation under investigation. The introduction of DG-DGGE allows one to unify run time conditions, independent of the retardation coefficient obtained by the Lerman program, resulting in an even improved resolution and rendering the technique highly suitable for routine work. It should be understood, however, that DG-DGGE does not per se modify the application of the Lerman program, since the choice of the denaturing gradient is still based on the melting map generated by this program on the basis of the specific sequence of each fragment under analysis. The denaturing gradient is chosen with the same criteria, i.e., by adopting, as the two extremes of the denaturing gradient, concentrations corresponding to values of $5^{\circ} \mathrm{C}$ above and $5^{\circ} \mathrm{C}$ below the $\mathrm{T}_{\mathrm{m}}$ values of the fragments under analysis. The only variable that we have modified is the migration time. Generally, with DGGE, the conditions chosen are $10 \mathrm{~V} / \mathrm{cm}$ with variable running times, depending on the retardation coefficient of the fragment under analysis. In our case, thus, for a 16-cm-long gel, one should adopt a total of $160 \mathrm{~V}$. However, with the present technique of DG-DGGE, one can adopt, for the different types of mutations, a standard running time which, for pure convenience, is set at $15 \mathrm{~h}$ (i.e., the typical length of an overnight run), while simultaneously reducing the voltage to about one-half the standard value. Such a standard running time, for widely different mutants exhibiting different retardation coefficients, can only be adopted in DG-DGGE, since the presence of the porosity gradient can further increase the mobility differences among annealed and partially unwound molecules, while maintaining the zone-sharpening effect. This could not possibly be achieved in conventional DGGE, since overrunning conditions would, in fact, decrease the mobility differences and lead to unacceptable zone diffusion. Of course, the user of the present technique can decide to still maintain the standard $10 \mathrm{~V} / \mathrm{cm}$ voltage gradient and adopt a shorter running time, according to his experimental needs. These novel experimental conditions can be found easily by analyzing a set of mutants under the standard DGGE in parallel with DG-DGGE.

\section{ACKNOWLEDGMENTS}

Supported in part by grants from the European Community (Biomed 2, Human Genome Project, BMH4-CT96- 
1158) to P.G.R. and by P.F. Ingegneria Genetica, to M.F. P.G.R. also gratefully acknowledges support from TelethonItaly (Grant N. E-153) and from AIRC (Associazione Italiana Ricerca sul Cancro).

\section{REFERENCES}

1.Brancolini, V., L. Cremonesi, E. Belloni, E. Pappalardo, R. Bordoni, M. Seia, S. Russo, R. Padoan, A. Giunta and M. Ferrari. 1995. Search for mutations in pancreatic sufficient cystic fibrosis Italian patients: detection of $90 \%$ of molecular defects and identification of three novel mutations. Hum. Genet. 96:312318.

2.Cheadle, J.P., E. Belloni, M. Ferrari, L. Millar-Jones and L. Millar-Jones. 1994. A novel mutation (M1V) in the translation initiation codon of the cystic fibrosis transmembrane conductance regulator gene, in three CF chromosomes of Italian origin. Hum. Mol. Genet. 3:1431-1432.

3.Claustres, M., B. Gerrard, M.B. White, M. Desgeorges, P. Kjiellberg, B. Rollin and M. Dean. 1992. A new mutation (1078delT) in exon seven of the CFTR gene in Southern French adult with cystic fibrosis. Genomics 13:907-908.

4.Cremonesi, L., M. Ferrari, E. Belloni, C. Magnani, M. Seia, P. Ronchetto, M. Rady, M.P. Russo, G. Romeo and M. Devoto. 1992. Four new mutations in the CFTR gene (541delC, R347H, R352Q, E585X) detected by DGGE analysis in Italian CF patients, associated with different clinical phenotypes. Hum. Mutation 1:314-319.

5.Cutting, G.R., L.M. Kasch, B.J. Rosenstein, J. Zielenski, L.-C. Tsui, S. E. Antonarakis and H.H. Kazazian Jr. 1990. A cluster of cystic fibrosis mutations in the first nucleotide binding folds of the cystic fibrosis conductance regulator protein. Nature 346:366-369.

6.Cutting, G.R., S.M. Curristan, E. Nash, B.J. Rosenstein, I. Lerer, D. Abeliovich, A. Hill, and C. Graham. 1992. Analysis of four diverse population groups indicates that a subset of cystic fibrosis mutations occurred in common among Caucasians. Am. J. Hum. Genet. 50:1185-1194.

7.Dean, M., M. White, J. Amos, B. Gerrard, C. Steward, K.-T. Khaw and M. Leppert. 1990. Multiple mutations in highly conserved residues are found in mildly affected cystic fibrosis patients. 1990. Cell 61:863-870.

8.Devoto, M., P. Ronchetto, P. Fanen, J.J. Telleria-Orriols, G. Romeo, M. Goossens, M. Ferrari, C. Magnani, M. Seia and L. Cremonesi. 1991. Screeening for non deltaF508 mutations in 5 exons of the CFTR gene in Italy. Am. J. Hum. Genet. 48:1127-1132.

9.Fisher, S.G. and L. Lerman. 1982. Analysis of point mutations by denaturing gradient gel electrophoresis. Proc. Natl. Acad. Sci. USA 80:1579-1583

10.Gasparini, P., V. Nunes, A. Savoia, M. Dognini, N. Morral, A. Gaona, A. Bonizzato, M. Chillon, F. Sangiuolo, G. Novelli, B. Dal- lapiccola, P.F. Pignatti and X. Estivill. 1991. The search for Southern European cystic fibrosis mutations: identification of two new mutations, four variants, and intron sequences. Genomics 10:193-200.

11.Gelfi, C., L. Cremonesi, M. Ferrari and P.G. Righetti. 1996. Temperature-programmed capillary electrophoresis for detection of DNA point mutations. BioTechniques 21:926-932.

12.Gianazza, E. and P.G. Righetti. 1979. Electrophoresis in gels of graded porosity and twodimensional techniques, p. 293-311. In P.G. Righetti, C.J. Van Oss and J.W. Vanderhoff (Eds.), Electrokinetic Separation Methods. Elsevier, Amsterdam.

13.Grompe, M. 1993. The rapid detection of unknown mutations in nucleic acids. Nature Genet. 5:111-117.

14.Guillermit, H., P. Fanen and C. Ferec. 1990. A $3^{\prime}$ splice site consensus sequence mutation in the cystic fibrosis gene. Hum. Genet. 85:450-453.

15.Kalin, A., T. Dork and B. Tummler. 1992. A cystic fibrosis allele encoding missense mutations in both nucleotide binding folds of the cystic fibrosis transmembrane conductance regulator. Hum. Mutation 1:204-210.

16.Kerem, B., J. Zielenski, D. Markiewicz, D. Bozon, E. Gazit, J. Jahaf, D. Kennedy, J.R. Riodran, F.S. Collins, J.R. Rommens and L.-C. Tsui. 1990. Identification of mutations in regions corresponding to the 2 putative nucleotide (ATP9-binding folds of the cystic fibrosis gene. 1990. Proc. Natl. Acad. Sci. USA 87:8447-8451.

17.Koch, M., R. Wahl and F.J. Seif. 1995. Analysis of thyroid stimulating hormone-receptor mutations by temperature gradient gel electrophoresis. Electrophoresis 16:742-743.

18.Menke, M.A.O.H., M. Tiemann, D. Vogelsang, C. Boie and R. Parwaresch. 1995. Temperature gradient gel electrophoresis for analysis of a PCR-based diagnostic clonality assay in the early stages of cutaneous T-cell lymphomas. Electrophoresis 16:733-738.

19.Myers, R.M., T, Maniatis and L.S. Lerman. 1987. Detection and localisation of single base changes by denaturing gradient gel electrophoresis. Methods Enzymol. 155:482-501.

20.Riesner, D., K. Henco and G. Steger. 1991. Temperature gradient gel electrophoresis: a method for the analysis of conformational transitions and mutations in nucleic acids and proteins. Adv. Electrophoresis 4:169-250.

21.Righetti, P.G. 1990. Immobilized pH Gradients: Theory and Methodology. Elsevier, Amsterdam.

22.Rothe, G.M. and W.D. Maurer. 1986. One dimensional PAA-gel electrophoretic techniques to separate functional and denatured proteins, p. 37-140. In M.J. Dunn (Ed.), Gel Electrophoresis of Proteins. Wright, Bristol.

23.Saba, L., G.B. Leoni, A. Meloni, V. Faà, A. Cao and M.C. Rosatelli. 1993. Two novel mutations in the transmembrane domain of the CFTR gene in subjects of Sardinian descent. Hum. Molec. Genet. 2:1739-1740.

24.Schlötterer, C. 1995. Temperature gradient gel electrophoresis as a screening tool for polymorphisms in multigene families. Electrophoresis 16:722-728.
25.Tiemann, M., M.A.O.H. Menke, R. Asbeck, H.H. Wacker and R. Parwaresch. 1995. Temperature gradient gel electrophoresis for analysis of clonal evolution in non-Hodgkin's lymphoma of the thyroid. Electrophoresis 16:729-732.

Received 16 January 1996; accepted 10 June 1996.

Address correspondence to:

Cecilia Gelfi

L.I.T.A.

Via Fratelli Cervi No. 93

Segrate 20090, Milano, Italy 